\title{
BMJ Open Baccalaureate nursing students' experiences with high-fidelity simulation: protocol for a qualitative systematic review
}

Yuxuan Zhu, Cong Geng (1) , Xianbo Pei, Xiaoli Chen

To cite: Zhu Y, Geng C, Pei X, et al. Baccalaureate nursing students' experiences with high-fidelity simulation: protocol for a qualitative systematic review. BMJ Open 2020;10:e040171. doi:10.1136/ bmjopen-2020-040171

- Prepublication history and additional material for this paper are available online. To view these files, please visit the journal online (http://dx.doi. org/10.1136/bmjopen-2020040171).

YZ and CG contributed equally.

YZ and CG are joint first authors.

Received 11 May 2020

Revised 14 September 2020

Accepted 26 October 2020
Check for updates

(C) Author(s) (or their employer(s)) 2020. Re-use permitted under CC BY-NC. No commercial re-use. See rights and permissions. Published by BMJ.

School of Health Sciences, Wuhan University, Wuhan, Hubei, China

\section{Correspondence to}

Xiaoli Chen;

chenx172@whu.edu.cn and

Xianbo Pei;

peixb@whu.edu.cn

\section{ABSTRACT}

Introduction High-fidelity simulation (HFS) can bridge the gap between theoretical knowledge and nursing practice and improve safety and quality of patient care in baccalaureate nursing education. Although inconsistent assessment instruments or lack of high-quality research designs affect the strength of the evidence and limit the generalisability of the results, quantitative studies generally demonstrate the effectiveness of HFS in baccalaureate nursing education. Synthesis of the existing evidence of baccalaureate nursing students' experiences with HFS is crucial for the improvement and revision of simulation design and teaching.

Methods and analysis A comprehensive search for qualitative studies on baccalaureate nursing students' experiences with HFS will be conducted in the following databases: PubMed, Embase, CINAHL, ProQuest, Web of Science, PsycINF0, the Cochrane library, China Biology Medicine disc, China National Knowledge Infrastructure and VIP Database for Chinese Technical Periodicals. This review considered studies reported in English or Chinese, and studies that were conducted between January 2000 and December 2019 in view of the launch of International Nursing Association for Clinical Simulation and Learning. The literature search will be conducted by two independent reviewers, and any disagreement will be adjudicated by discussion or with a third reviewer. The two independent reviewers will use the Joanna Briggs Institute (JBI) Critical Appraisal Checklist for Qualitative Research to assess the methodological validity, following which the JBI standardised data extraction tools will be used to extract relevant data. The JBI meta-aggregation method will be subsequently used to synthesise the data, eventually forming themes, categories and synthesised findings. The final synthesised findings will establish confidence levels based on the JBI ConQual approach.

Ethics and dissemination This review does not require formal ethical review since it is based on available published literature. Findings will be disseminated through publication in a peer-reviewed journal, and, if possible, presented in scientific conferences.

\section{INTRODUCTION}

With rapid changes in the clinical environment, especially regarding patient safety concerns, ethical issues and clinical practice
Strengths and limitations of this study

- Some scholars have examined students' experience with high-fidelity simulation (HFS) using qualitative research methods over the last few years.

- This review is the first qualitative synthesis focused on baccalaureate students' experiences of HFS.

- Obtaining a deeper understanding of students' experiences with HFS-based learning is crucial to improving simulation design and teaching.

- This protocol minimises the bias of analysis of qualitative studies using the Joanna Briggs Institute qualitative systematic review method following the Enhancing Transparency in Reporting the Synthesis of Qualitative Research Statement guidelines.

- As a qualitative systematic review, the findings are limited by the quality and context of included original studies.

arrangement, opportunities for nursing students to handle problem-based clinical situations and direct experience with patient care have diminished. ${ }^{1}{ }^{2}$ Nursing educators have explored innovative pedagogical approaches to prepare undergraduate nursing students to adapt to the rapidly changing clinical environment. ${ }^{3}$ As an effective learning and teaching strategy, use of simulation in nursing education has exponentially increased, thereby providing nursing students with a safe and realistic environment to practice without any concerns regarding patient safety. ${ }^{45}$ Simulation, broadly defined, includes standardised patients, low-fidelity simulation, medium-fidelity simulation and high-fidelity simulation (HFS) ${ }^{6}$ HFS provides a near-real experience, including realistic physiological responses, the ability to interact and communicate with the mannequin and other feedback mechanisms, ${ }^{7}$ thereby improving students' experience and satisfaction with simulation-based learning. HFS provides students with practical 'hands-on' application of theoretical knowledge, thereby 
bridging the gap between theoretical knowledge and the current demands of nursing practice, and improving safety and quality of patient care. ${ }^{78}$ Therefore, HFS has gained popularity in nursing education programmes.

A large-scale study conducted by the National Council of State Boards of Nursing of the USA shows that simulation can safely replace up to $50 \%$ of clinical practice time without affecting the learning effect and the ability of nursing students, and suggested that it should be increasingly applied in nursing education. ${ }^{9}{ }^{10}$ Previous quantitative studies of HFS provide substantial evidence supporting cognitive, emotional and psychomotor learning outcomes. ${ }^{11} 12$ Specifically, nursing students' knowledge acquisition, ${ }^{13}$ clinical skill competence, ${ }^{1415}$ critical thinking, ${ }^{16}$ clinical reasoning, ${ }^{17}$ clinical judgement, ${ }^{18}$ communication skills, ${ }^{19}$ confidence, ${ }^{131416}$ self-efficacy $^{20}$ and motivation ${ }^{21}$ can be potently augmented through HFS. Nevertheless, various assessment instruments used for the same test purpose in quantitative research limit further comparison. ${ }^{22}$ Overall, the lack of high-quality study designs lowers the credibility of existing evidence. Therefore, the current quantitative research outcomes should be regarded with some caution. ${ }^{22} 23$

Most studies on simulation-based learning among baccalaureate nursing students are primarily based on quantitative designs, which do not examine students' experience as a crucial element influencing simulationbased learning. ${ }^{24-26}$ However, baccalaureate nursing students' experiences with HFS are critical factors that should be considered for improvement and revision of simulation design and teaching. ${ }^{27} 28$ Some scholars have examined students' experience with HFS using qualitative research methods over the last few years. Nevertheless, the students' experiences are various because of different perspectives and simulation curriculum designs. ${ }^{29}{ }^{30} \mathrm{~A}$ systematic synthesis of qualitative studies of baccalaureate nursing students' experience with HFS could reveal common themes, leading to the generation of knowledge that may not be revealed by a single study. In addition, a qualitative systematic review could obtain a comprehensive understanding of the diverse experience of baccalaureate nursing student, and provide strong, reliable, and significant evidence for the development of simulation-based learning.

An initial search in the Joanna Briggs Institute (JBI) Database of Systematic Reviews and Implementation Reports and the Cochrane Database of Systematic Reviews revealed no relevant systematic review that explored baccalaureate nursing students' experiences with HFS. Only one qualitative systematic review focused on students' and educators' experiences of maternal and child simulation-based learning. ${ }^{31}$ In addition, this review specifically focused on integrating experiences in maternal and child simulation-based learning activities in undergraduate nursing education programmes. A more comprehensive understanding of students' experience, including all disciplines of HFS, should be considered. Thus, a qualitative systematic review of published qualitative studies of HFS is warranted to improve and revise simulation design and teaching in baccalaureate nursing education. We have registered this review in the JBI System.

\section{Objectives}

The purpose of this systematic review is to synthesise published evidence exploring baccalaureate nursing students' experiences with HFS in educational settings. This review may provide insights into the students' experience with simulation-based learning while identifying barriers and enablers in simulation-based learning. The robust and multidimensional evidence integrated from original qualitative studies can further provide references for nursing educators to make well-informed decisions while developing strategies targeted to optimise students' learning experience with simulation.

The research questions are as follows:

1 . What are the experiences of baccalaureate or undergraduate nursing students with HFS?

2. What are the barriers and enablers to the use of HFS in baccalaureate or undergraduate nurse education?

\section{METHODS AND ANALYSIS \\ Inclusion criteria \\ Participants}

This systematic review will consider studies that include baccalaureate or undergraduate nursing students.

\section{Phenomena of interest}

This systematic review will include studies that explore the experiences of undergraduate or baccalaureate nursing students with HFS. Moreover, this review will consider all types of HFS, including but not limited to high-fidelity patient simulation, high-fidelity human simulation, human patient simulator and human patient simulation.

\section{Context}

This review will consider studies that use HFS in undergraduate nursing education.

\section{Types of studies}

This study will include qualitative research studies focusing on students' experiences with HFS-based learning, including but not limited to phenomenology, action research, grounded theory, feminism research and ethnography research. This study will also consider mixed-methods studies aimed at understanding students' experience with HFS. Further, this systematic review will consider studies published in English or Chinese.

\section{Search strategy}

We propose to use a pre-planned sensitive search strategy that aims to find both published and grey literature. First, we will conduct a preliminary search of the PubMed and CINAHL databases to analyse the keywords and determine the index terms. A tailored search strategy will be used to search various databases to ensure that all available 
studies are retrieved. The specified search term will then be developed and used to search the following databases: PubMed, ProQuest, the Cochrane Library, CINAHL, Embase, Web of Science, PsycINFO, China Biology Medicine disc, China National Knowledge Infrastructure and VIP Database for Chinese Technical Periodicals. The search for grey literature would include the following sources: The International Nursing Association for Clinical Simulation and Learning (INACSL), ProQuest Dissertations and Theses, Open Grey collection and Deep Blue Library databases. The reference list of all the identified studies will be searched to identify other relevant studies. Initial keywords to be used are: simulation*, undergraduate or baccalaureate nursing students; qualitative*. The customised search strategy used in PubMed is shown in online supplemental file 1 .

Considering the advances in simulation technology, studies published from January 2000 to December 2019 will be included in this review, in view of the launch of the INACSL. ${ }^{32}$

\section{Study records}

We will use NoteExpress V.3.2.0 software for document management, and the documents screened and selected in each step will be managed and recorded through subsequent NoteExpress databases.

\section{Study selection}

Two reviewers will independently evaluate the records (YZ and CG). Following the comprehensive search of the database, all identified citations will be imported to NoteExpress V.3.2.0 software. Two independent reviewers will screen the titles and abstracts according to the inclusion criteria. The studies that meet the inclusion criteria will be selected for a detailed evaluation of the full text based on the inclusion criteria. Further, all potential studies will be examined carefully by two independent reviewers. The eligible studies and their details will be collated and uploaded into a new NoteExpress database, which will undergo a process of critical appraisal. To maintain consistency, the two reviewers will converse regularly, and any disagreement will be adjudicated by a third reviewer.

\section{Assessment of methodological quality}

The methodology of the selected studies will be screened and assessed by two independent reviewers (YZ and CG), using the JBI's instrument of Critical Appraisal Checklist for Qualitative Research, prior to inclusion. The checklist includes 10 items that assess different domains, including research methodology, philosophical foundation, data collection, analysis method and result validity. ${ }^{33}$ All items will be evaluated by 'yes', 'no' and 'unclear'. The evaluation results are judged by the number of items that meet the standard requirements. A rating of $\leq 6$ is considered weak, $7-8$ is considered medium, and $9-10$ is considered strong. ${ }^{34}$ Any disagreements that arise between reviewers will be adjudicated by a third reviewer until three reviewers come to a consensus. The results of the critical appraisal checklist will be reported with an overall rating, and only studies with at least a medium rating will undergo data extraction and synthesis (online supplemental file 2).

\section{Data extraction}

Two independent reviewers ( $\mathrm{YZ}$ and $\mathrm{CG}$ ) will extract qualitative data related to research questions and objectives from the included studies, using the JBI Qualitative Assessment and Review Instrument Data Extraction Tools for Qualitative Research (online supplemental file 3). ${ }^{33}$ The following specific information will be extracted from included studies: study objectives; pedagogical approach and content; study design: methodological, philosophy underpinnings, sample size and setting; study method: data collection and analysis techniques. The two reviewers (YZ and CG) will then enter all the text under the headings 'results/conclusions' into Microsoft Word software and assign a level of credibility for extracted findings.

\section{Data synthesis}

The extracted data will be analysed and synthesised using the JBI meta-aggregation method, which enables generalisable statements in the form of recommendations to guide practitioners and policy-makers. ${ }^{33}{ }^{35}$ The two independent reviewers (YZ and CG) will read the full text of included studies verbatim to obtain a preliminary understanding. The reviewers will then assemble and rate the quality of the extracted findings into three levels: unequivocal, equivocal and unsupported. Further, the findings will be coded line by line to draw meaningful concepts that be consistent with the meaning of original context. Reviewers will derive themes on the basis of similarity in meaning and subject categories to a meta-synthesis to produce a single comprehensive set of synthesised findings for better educational practice. Furthermore, two reviewers (XC and $\mathrm{XP}$ ) will resolve disagreements, if any, surrounding data synthesis by discussion and finally form the theme catalogue.

\section{Assessing the certainty of findings}

To establish the confidence level, the synthesised findings will be rated as high, moderate, low or very low based on the JBI ConQual approach (online supplemental file 4), which appraises dependability and credibility. Two reviewers (YZ and CG) will independently complete this form, and any disagreement will be resolved by discussion. ${ }^{36}$

\section{Reporting of protocol}

This qualitative meta-synthesis review will adopt the JBI meta-aggregative approach following the Enhancing Transparency in Reporting the Synthesis of Qualitative Research Statement ${ }^{37}$ and will be carried out from June to August 2020. The qualitative systematic review protocol is conducted based on the Preferred Reporting Items for Systematic Reviews and Meta-analyses Protocols checklist to ensure rigour in research. ${ }^{38}$ The relevant items are included in online supplemental file 5 . 


\section{Patient and public involvement}

Patients and the public were not involved in the preparation of this protocol and will not be directly involved in the final systematic review.

\section{ETHICS AND DISSEMINATION}

As the published original literature requires ethical review, this systematic review does not require formal ethical considerations. If possible, our findings will be published in a peer-reviewed journal or presented in scientific conferences. The final published article will report any changes and revisions arising from the protocol.

\section{Twitter Yuxuan Zhu @zhuyuxuan}

Acknowledgements We would like to thank Yan Wang, an expert librarian, for helping us with the development of the search strategy for this systematic review.

Contributors Study conception and design, literature search, data extraction and appraisal, data synthesis and interpretation, manuscript drafting: YZ and CG; data selection, data appraisal, data synthesis, manuscript critical revision, and arbitrate in cases of disagreement: XC and XP. All the authors have read, provided feedback, and approved the final manuscript. YZ and CG contributed equally.

Funding This work was supported by the Wuhan University Teaching and Research Reform Project grant number 2018JG054, and the Wuhan University Medical Department Teaching and Research Reform Project grant number 2019070.

Competing interests None declared.

Patient consent for publication Not required.

Provenance and peer review Not commissioned; externally peer reviewed.

Supplemental material This content has been supplied by the author(s). It has not been vetted by BMJ Publishing Group Limited (BMJ) and may not have been peer-reviewed. Any opinions or recommendations discussed are solely those of the author(s) and are not endorsed by BMJ. BMJ disclaims all liability and responsibility arising from any reliance placed on the content. Where the content includes any translated material, BMJ does not warrant the accuracy and reliability of the translations (including but not limited to local regulations, clinical guidelines, terminology, drug names and drug dosages), and is not responsible for any error and/or omissions arising from translation and adaptation or otherwise.

Open access This is an open access article distributed in accordance with the Creative Commons Attribution Non Commercial (CC BY-NC 4.0) license, which permits others to distribute, remix, adapt, build upon this work non-commercially, and license their derivative works on different terms, provided the original work is properly cited, appropriate credit is given, any changes made indicated, and the use is non-commercial. See: http://creativecommons.org/licenses/by-nc/4.0/.

ORCID iD

Cong Geng http://orcid.org/0000-0002-9122-248X

\section{REFERENCES}

1 Kim J, Park J-H, Shin S. Effectiveness of simulation-based nursing education depending on fidelity: a meta-analysis. BMC Med Educ 2016;16:152.

2 Small SP, Colbourne PA, Murray CL. High-Fidelity simulation of pediatric emergency care: an eye-opening experience for Baccalaureate nursing students. Can J Nurs Res 2018;50:145-54.

3 Hickey MT. Baccalaureate nursing graduates' perceptions of their clinical instructional experiences and preparation for practice. J Prof Nurs 2010;26:35-41.

4 King M. Developing a high-fidelity simulation program in a nursing educational setting. Health Care Manag 2018;37:235-49.

5 Lestander Örjan, Lehto N, Engström Åsa. Nursing students' perceptions of learning after high fidelity simulation: effects of a three-step Post-simulation reflection model. Nurse Educ Today 2016;40:219-24.

6 Olson JK, Paul P, Lasiuk G, et al. The state of knowledge regarding the use of simulation in Pre-Licensure nursing education: a mixed methods systematic review. Int J Nurs Educ Scholarsh 2018;15. doi:10.1515/ijnes-2017-0050. [Epub ahead of print: 20 Feb 2018].

7 Massoth C, Röder H, Ohlenburg H, et al. High-fidelity is not superior to low-fidelity simulation but leads to overconfidence in medical students. BMC Med Educ 2019;19:29.

8 Fawaz MA, Hamdan-Mansour AM. Impact of high-fidelity simulation on the development of clinical judgment and motivation among Lebanese nursing students. Nurse Educ Today 2016;46:36-42.

9 Hayden JK, Smiley RA, Gross L. Simulation in nursing education: current regulations and practices. J Nurs Regul 2014;5:25-30:25-30.

10 Kaddoura M, Vandyke O, Smallwood C, et al. Perceived benefits and challenges of repeated exposure to high fidelity simulation experiences of first degree accelerated bachelor nursing students. Nurse Educ Today 2016;36:298-303.

11 Vincent MA, Sheriff S, Mellott S. The efficacy of high-fidelity simulation on psychomotor clinical performance improvement of undergraduate nursing students. Comput Inform Nurs 2015;33:78-84.

12 La Cerra C, Dante A, Caponnetto V, et al. Effects of high-fidelity simulation based on life-threatening clinical condition scenarios on learning outcomes of undergraduate and postgraduate nursing students: a systematic review and meta-analysis. BMJ Open 2019;9:e025306.

13 Crowe S, Ewart L, Derman S. The impact of simulation based education on nursing confidence, knowledge and patient outcomes on general medicine units. Nurse Educ Pract 2018;29:70-5.

14 Kiernan LC. Evaluating competence and confidence using simulation technology. Nursing 2018;48:45-52.

15 Lejonqvist G-B, Eriksson K, Meretoja R. Evidence of clinical competence by simulation, a hermeneutical observational study. Nurse Educ Today 2016;38:88-92.

16 Alamrani MH, Alammar KA, Alqahtani SS, et al. Comparing the effects of simulation-based and traditional teaching methods on the critical thinking abilities and Self-Confidence of nursing students. J Nurs Res 2018;26:152-7.

17 Bae J, Lee J, Jang Y, et al. Development of simulation education Debriefing protocol with faculty guide for enhancement clinical Reasoning. BMC Med Educ 2019;19:197.

18 Yang F, Wang Y, Yang C, et al. Improving clinical judgment by simulation: a randomized trial and validation of the Lasater clinical judgment rubric in Chinese. BMC Med Educ 2019;19:20.

19 MacLean S, Kelly M, Geddes F, et al. Use of simulated patients to develop communication skills in nursing education: an integrative review. Nurse Educ Today 2017;48:90-8.

20 Jonson C-O, Pettersson J, Rybing J, et al. Short simulation exercises to improve emergency department nurses' self-efficacy for initial disaster management: controlled before and after study. Nurse Educ Today 2017;55:20-5.

21 Jeppesen $\mathrm{KH}$, Christiansen S, Frederiksen K. Education of student nurses - A systematic literature review. Nurse Educ Today 2017:55:112-21.

22 Cant RP, Cooper SJ. Use of simulation-based learning in undergraduate nurse education: an umbrella systematic review. Nurse Educ Today 2017;49:63-71.

23 Cant RP, Cooper SJ. The value of simulation-based learning in pre-licensure nurse education: a state-of-the-art review and metaanalysis. Nurse Educ Pract 2017;27:45-62.

24 Shin S, Park J-H, Kim J-H. Effectiveness of patient simulation in nursing education: meta-analysis. Nurse Educ Today 2015;35:176-82.

25 Najjar RH, Lyman B, Miehl N. Nursing students' experiences with high-fidelity simulation. Int J Nurs Educ Scholarsh 2015;12. doi:10.1515/ijnes-2015-0010. [Epub ahead of print: 19 Mar 2015].

26 Akselbo I, Olufsen V, Ingebrigtsen $\mathrm{O}$, et al. Simulation as a learning method in public health nurse education. Public Health Nurs 2019;36:226-32.

27 Kolb D. Experiential learning: experience as the source of learning and development. Pearson Schweiz Ag 1983;1:16-17.

28 MacLean S, Geddes F, Kelly M, et al. Realism and presence in simulation: nursing student perceptions and learning outcomes. J Nurs Educ 2019;58:330-8.

29 Chiang VCL, Chan SSC. An evaluation of advanced simulation in nursing: a mixed-method study. Collegian 2014;21:257-65.

30 Roy L. Baccalaureate nursing students' perceptions of simulation and the development of clinical judgment, 2014.

31 MacKinnon K, Marcellus L, Rivers J, et al. Student and educator experiences of maternal-child simulation-based learning: a systematic review of qualitative evidence protocol. JBI Database System Rev Implement Rep 2015;13:14-26. 
32 Bland AJ, Topping A, Wood B. A concept analysis of simulation as a learning strategy in the education of undergraduate nursing students. Nurse Educ Today 2011;31:664-70.

33 Aromataris E, Munn Z. Joanna Briggs Institute reviewer's manual. The Joanna Briggs Institute, 2017.

$34 \mathrm{He} \mathrm{J}$, Wang Y, Liu Y, et al. Experiences of pregnant women with gestational diabetes mellitus: a systematic review of qualitative evidence protocol Joanna Briggs Institute reviewer's manual. BMJ Open 2020;10:e034126. doi:10.1136/bmjopen-2019-034126

35 Lockwood C, Munn Z, Porritt K. Qualitative research synthesis: methodological guidance for systematic reviewers utilizing metaaggregation. Int J Evid Based Healthc 2015;13:179-87.
36 Munn Z, Porritt K, Lockwood C, et al. Establishing confidence in the output of qualitative research synthesis: the ConQual approach. BMC Med Res Methodol 2014;14:108.

37 Tong A, Flemming K, Mclnnes E, et al. Enhancing transparency in reporting the synthesis of qualitative research: ENTREQ. BMC Med Res Methodol 2012;12:181.

38 Moher D, Shamseer L, Clarke M, et al. Preferred reporting items for systematic review and meta-analysis protocols (PRISMA-P) 2015 statement. Syst Rev 2015;4:1. 